\title{
The silent treatment
}

\author{
Biotech firms are vying to harness the potential of RNA interference. But will its impact \\ be in finding new disease targets, or in RNA-based drugs? Julie Clayton investigates.
}

T he hype surrounding RNA interference (RNAi) 'gene-silencing' technology has both academic labs and biotech companies firmly in its grip. RNAi is lauded as a powerful approach to gene control, with the promise of revolutionizing basic research and providing treatments for intractable conditions such as cancer and neurodegenerative disease.

Far from being an established tool, RNAi is still a maturing technology with some way to go before its true potential is known. Many in the field are divided over whether its greatest contribution will lie in the identification and validation of disease targets - for new drugs ranging from small-molecule inhibitors to antibodies - or in the less certain development of RNAi itself as a therapeutic.

Until relatively recently, RNAi was the preserve of those studying plants and invertebrates, notably the nematode Caenorhabditis elegans. But three years ago Tom Tuschl and his colleagues, then at the Max Planck Institute for Biophysical Chemistry in Göttingen, Germany, showed that the technology could be used reliably to silence gene expression in mammalian cells (S. M. Elbashir et al. Nature 411, 494-498; 2001). "It took everybody by surprise", says Nassim Usman, senior vice-president of Sirna Therapeutics in Boulder, Colorado, one of the firms aiming to develop RNAi as a therapy.

RNAi relies on the introduction of double-stranded RNA mol ecules into cells to block the translation of messenger RNAs into protein. The double-stranded RNA, which must be partly identi$\mathrm{cal}$ in sequence to the gene to be inhibited, is cleaved into shorter fragments in the cell by an enzyme called Dicer, and the 'sense' strand degraded. The remaining 'antisense' strand then becomes incorporated into a protein complex called RNA-induced silencing complex. The activity of this complex is directed by the RNA towards the target messenger RNA, which is degraded, thus effectively 'silencing' the gene from which it was transcribed (C. D. Novina and P. A. Sharp Nature 430, 161-168, 2004; G. J. Hannon Nature 418, 244-251, 2002; and Nature, 431,337-378, 2004).

Key to Tuschl's success was the discovery that if the introduced RNAs were short enough - between 21 and 23 nucleotides long - they would not trigger the mammalian cell's defence mechanism against RNA viruses, the production of interferon, which was masking the effect of gene silencing. Attention turned to these short RNAs, called small interfering RNAs (siRNA), which can be synthesized chemically to match the desired target gene.

A slew of studies then showed that RNAi was an impressive tool for manipula ting genes in laboratorycultured mammalian cells, raising the tantalizing prospect of doing the same in animal models, and possibly even in humans. With impressive speed, life-sciences equipment supply companies have developed kits and reagents for supporting RNAi-based research (see 'RNAi options', below). At the same time, new companies have emerged, hungry for partners, offering large-scale and high-throughput RNAibased analysis of gene function, for both basic research and for the identification and validation of new drug targets. "RNAi has turned out to be a wonderful target-validation tool", says Dimitry Samarsky, head of business and technology development at California-based Invitrogen.

\section{RNAi OPTIONS}

Kits and reagents for making the various types of RNA interference (RNAi) constructs and getting them into cells are now widely available. The simplest options are designed to enable the negatively charged, chemically synthesized small interfering RNA (siRNA) molecules to enter cells more easily. One way is to use electroporation. Alternatively, the siRNA can be encapsulated in lipids or polymers to overcome the repulsive charges of the cell membrane, as in kits supplied by companies such as Ambion in Austin,

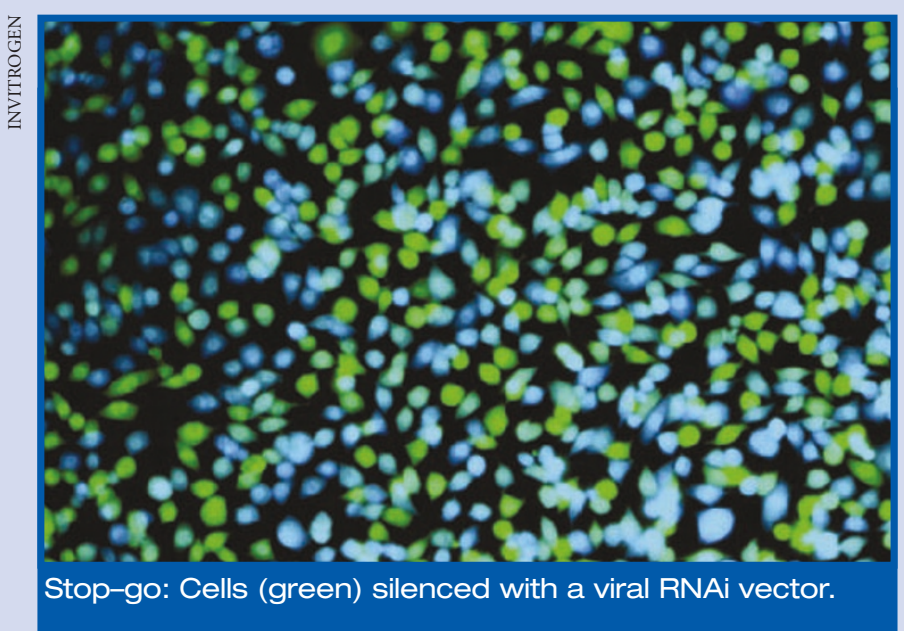

Texas, Invitrogen in Carlsbad, California, and QIAGEN in Venlo, the Netherlands. QIAGEN has developed siRNA sets against specific functional gene families and pathways, such as those leading to apoptosis and cancer. Their Human Druggable Genome siRNA Set targets 5,000 human genes of therapeutic interest. For high-throughput siRNA-based screening, QIAGEN offers an RNAi human/mouse control kit that provides all the reagents necessary for successful start-up. Oligonucleotide producers now also make siRNAs or their DNA equivalents to order, and siRNA manufacturers such as Dharmacon in Dallas, Texas, MWG Biotech in Ebersberg, Germany, and Proligo in Boulder, Colorado, offer free online design services.

Transfected siRNAs achieve significant gene 'knock-down' for some 3-7 days before being naturally degraded. This is usually sufficient for studying the immediate effects of inhibiting gene expression, when screening for drug targets, for example, but is likely not to be sufficient for RNAi-based therapy.

An effect lasting for weeks can be obtained with expression systems based on plasmid or viral vectors carrying DNA versions of the interfering RNA. The DNA is transcribed and the transcript processed into siRNA within the cell. In many of these systems the constructs are expressed as short double-stranded RNA 'hairpins' (shRNA), which are then cleaved by cellular enzymes to produce functional siRNAs. Vectors based on adenoviruses and lentiviruses also enable RNAi to be extended to a wider range of non-dividing primary cells than when transfecting with siRNA or a plasmid. Kits for constructing adenoviral and lentiviral vector RNAi expression systems are supplied by Promega in Madison, Wisconsin, BD Biosciences in San Jose, California, Imgenex in San Diego, California, Ambion and Invitrogen. 


\section{From worms to humans}

Cenix BioScience in Dresden, Germany, is one of the new firms that has been formed to seize these opportunities. Chief executive Christophe Echeverri felt nervous about leaving academia as a postdoc to start up the company in 1999, but has never looked back. Cenix is developing RNAi technology for basic research and drug-target validation, and mainly offers in vitro screening of cell lines using a library of siRNAs designed against the human genome, and against potentially druggable targets such as kinases (see Nature 428, 225-231; 2004). siRNAs "really represent the gold standard reagent with the broadest applicability" compared with vectorexpressed short hairpin RNA (shRNA), says Echeverri. The level of gene silencing produced with shRNA is more variable, less reproducible, and does not permit control over the dose achieved inside cells, he says.

"Synthetic siRNA is the preferred choice for high-throughput screening," agrees Walter Tian, business director at QIAGEN, based in Venlo, the Netherlands. Unlike siRNA, he adds, the vector-based shRNA approach still faces challenges, such as the need to create effective design algorithms and the generation of genome-wide libraries. The need for individual optimization means that the shRNA approach may not be cost-effective for high-volume labs that simply want to screen easily transfected cultured cell lines.

A not-for-profit organization, the Translational Genomics Research Institute in Gaithersburg, Maryland, carries out con-

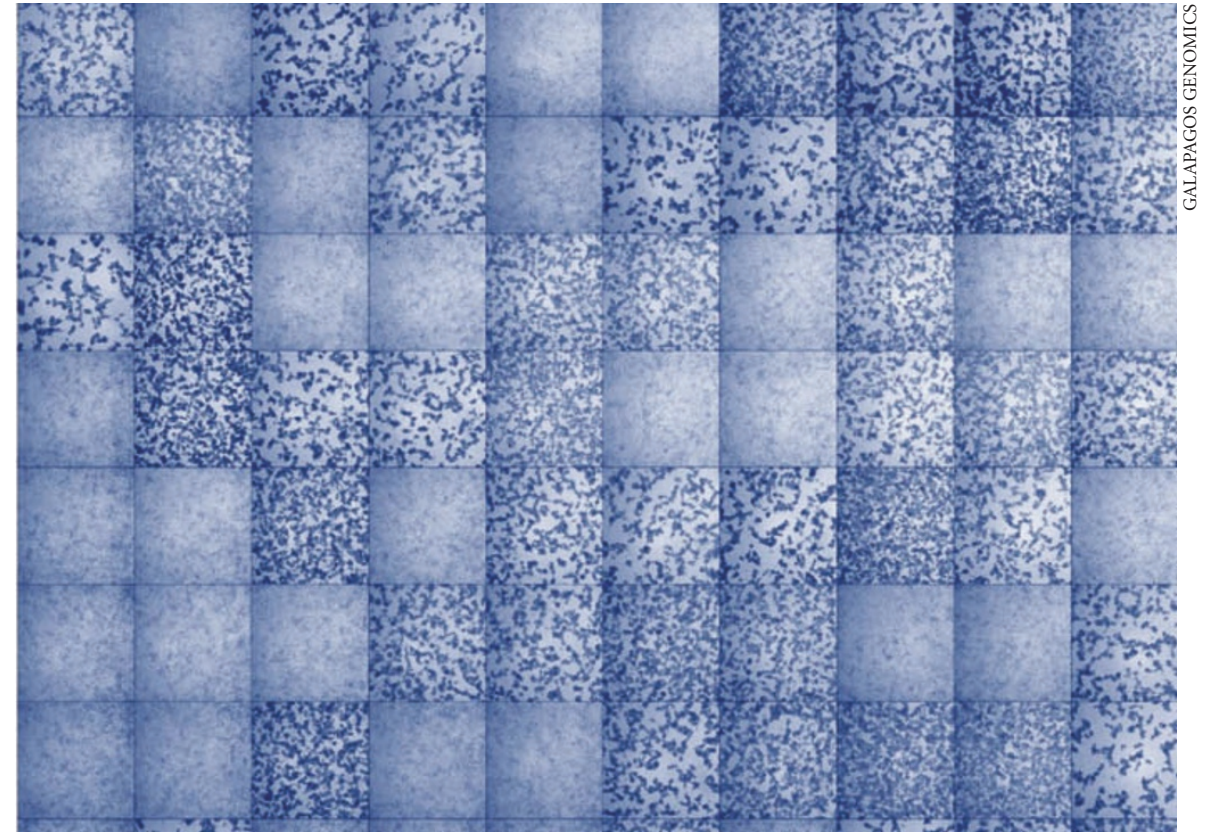

Scaling up: shRNA knock-down of gene expression in a microtitre plate.

tract screening of cell lines and primary cells for targets related to cancer biology, using QIAGEN's library of 10,000 siRNAs against 5,000 druggable targets, and about 25,000 shRNA vectors. It looks for "points of vulnerability" - new drug targets and ways to enhance the effectiveness of existing drugs, says Spyro Mousses, director of the institute's cancer drug development laboratory.

In contrast to the siRNA approach, Belgium-based biotech company Galapagos
Genomics specializes in high-throughput screening of human primary cells using proprietary adenovirus vectors expressing shRNAs against 4,900 known druggable targets involved in a range of diseases, including rheumatoid arthritis, osteoarthritis, asthma and Alzheimer's disease. The viral vectors have modified protein coats to broaden the target-cell range, and are arrayed in 96- or 384-well plates to give relatively highthroughput screening. Galapagos is also

\section{EXPRESS DELIVERY}

At the University of California, Los Angeles, professor of medicine William Pardridge has developed a 'molecular Trojan horse' strategy for RNA interference (RNAi) delivery, which is licensed to drug-delivery specialists ArmaGen Technologies in Santa Monica, California. It consists of incorporating either peptides or monoclonal antibodies that target membrane-bound receptors into 85-nanometre-diameter liposomes containing plasmid DNA with the desired siRNA sequence. The lipids are coupled to polyethylene glycol to prevent nonspecific fusion with the cell membranes of non-target cells. This strategy aims both to protect the DNA from degradation by nucleases and to

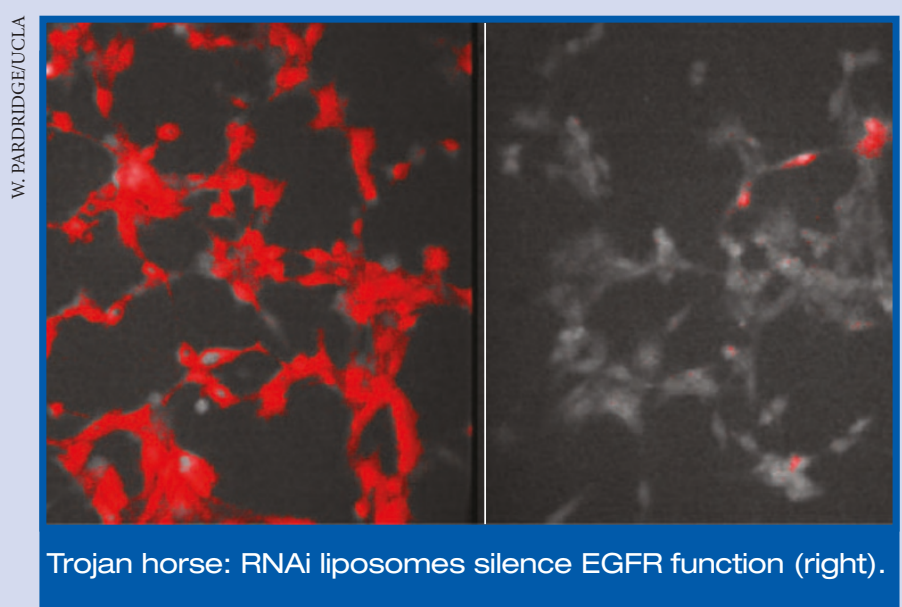

ensure its sequential uptake by endocytosis across the blood-brain barrier and the cell and nuclear membranes of neurons. The system has been tested in adult mice and rhesus monkeys, and Pardridge hopes to see it in clinical trials against brain cancer "within two years".

Trojan horses also feature in TargeTran, the delivery system from Intradigm in Rockville, Maryland. TargeTran involves nanoparticles formed by the selfassembly of positively charged polymers with the negatively charged siRNA, encasing and protecting it. Additional ligands are added for targeting to particular tissues after intravenous injection, and combinations of siRNAs can be used to inhibit multiple drug targets.

One of Intradigm's targets is the vascular endothelial growth factor (VEGF) pathway, which is involved in angiogenesis in tumours and other diseases. Martin Woodle, chief scientific officer at Intradigm, hopes to take the VEGFtargeting technology into the clinic, first for cancer next year, and then against age-related macular degeneration, a relatively common cause of blindness, which involves abnormal blood vessel growth in the retina. Alnylam, of Cambridge, Massachusetts, is also developing siRNA therapeutics against this condition.

But Woodle's collaborator Raymond Schiffelers at the Netherlands Cancer Institute in Amsterdam cautions that many ligands targeting multiple receptors are likely to be needed for the clinical application of RNAi against cancer, which in humans "will be far more complex because there are different phases of tumorigenesis where parts of the process are not angiogenic". Intradigm is also collaborating with researchers in Guang Zhou, Hong Kong and Beijing to produce RNAi-based therapy against the coronavirus that causes severe acute respiratory syndrome (SARS). 
developing animal models for in vivo target validation using shRNA, expressed from an adenovirus vector, says cellular and molecular biology director Helmuth van Es - for example, to target the synovium in a collageninduced model of arthritis.

High-throughput screening has revealed two bottlenecks in the target-discovery process, according to Sumit Chanda at the Genomics Institute of the Novartis Research Foundation (GNF) in San Diego, California. The first is the need to make RNAi screening in primary cell cultures as fast and efficient as in cell lines. The second is to translate in vitro results into animal models.

siRNA delivery to primary cells remains less efficient than in transformed cell lines, and not every primary cell type is amenable to lipid-based transfection. As an alternative, a few companies, including BTX in Holliston, Massachusetts, and Cyto Pulse Sciences in Columbia, Maryland, offer 96-well platebased electroporation. The GNF is developing high-throughput electroporation that would enable transfection with 20,000 oligonucleo-tides at a time, says Chanda, but this is not yet commercially available. And attempts to use vectors to deliver individual inhibitory RNAs to 20,000 wells at a time harbour significant technical and safety challenges, Chanda adds, including the need to achieve even titres of virus per well and to conduct all procedures to appropriate biosafety standards.

\section{Down but not out}

Several groups are tackling the second bottleneck - in vivo validation. Compared with the creation of gene knockouts in mice, which can take up to nine months, RNAi in animals can potentially produce answers within days or weeks, greatly speeding up the number of drug targets that can be validated in a given time. The often incomplete
nature of RNAi - usually up to $90 \%$ knock-down - means that it may reflect more accurately the situation in human disease, where a disease-causing gene may be operating at suboptimal levels. It also mimics more closely the effect of a smallmolecule drug, which usually achieves only incomplete inhibition of its target.

Effective RNAi in vivo in animals could mean vast savings for the drug industry, says Martin Woodle of siRNA therapeutics company Intradigm in Rockville, Maryland. Traditionally it can cost from US\$10 million to $\$ 50$ million to identify a new small-molecule drug and go to the first tests in animals, making it expensive if drug targets that looked promising in vitro fail at this stage. Further savings will occur if the siRNA itself can be developed as the drug.

For research purposes, the GNF is trying to take RNAi into vertebrates with faster generation times than mice, such as zebrafish and chicks. But most research groups and companies are concentrating on developing the use of RNAi in mouse models of disease.

\section{Getting it there}

The key challenge for achieving effective RNAi in vivo is delivery to the desired organ and into the target cells, to ensure specificity and adequate dose. "RNAi will never leave the Petri dish until we solve the delivery problems," says William Pardridge, professor of medicine at the University of California, Los Angeles. But it's good news for the biotech sector that there is unlikely to be a single solution. "I think the delivery problem will be solved - either by delivery locally, into the local blood supply, or by targeting," says Judy Lieberman, a paediatrician at Harvard University's CBR Institute for Biomedical Research. "Viral vectors may make more sense for genetic diseases when you want long-term efficacy. There are so many possible indications that some delivery methods may be better than others."

One approach is to target the RNAi construct specifically at a tissue. Either the siRNA or the DNA encoding it is encased in a liposome or polymer coat, and ligands are incorporated into the coat that bind to cellsurface receptors on the target tissue, which

\section{A RIVAL TO ANTISENSE?}

Alongside the optimism over RNA interference (RNAi) comes the inevitable question of whether it will fare better than previous RNA-based technologies

- antisense and ribozymes - both in the laboratory and in the clinic.

Many commentators are placing their bets on RNAi because it taps into a pre-existing control system within the cell and has the potential for greater potency, given that the same small interfering RNA (siRNA) molecules can recycle between different copies of messenger RNA. Single-stranded antisense molecules do not exist naturally, and each one acts only once to block translation of messenger RNA before being degraded. Ribozymes do cleave multiple copies of the same messenger RNA, but their potency remains in question, and there is, as yet, no sign of success as potential therapeutics.

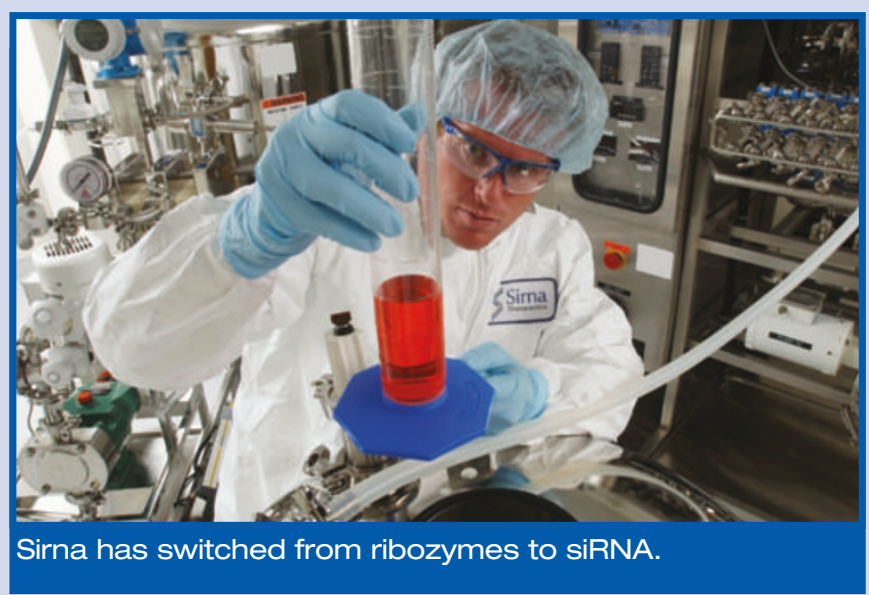

"RNAi is what antisense never was. It's robust and reproducible, and it exists in nature," says Inder Verma of the Salk Institute for Biological Sciences in San Diego, California. RNAi is "much more active and effective than antisense and ribozymes", according to Martin Woodle of Intradigm in Rockville, Maryland.

Frank Bennett of Isis Pharmaceuticals in Carlsbad, California, disputes this, at least for in vitro use, saying that "by and large antisense and RNAi are equally potent in cell culture". For in vivo use, he asserts that RNAi has yet to be fully optimized to justify comparison with antisense. "We're keeping an open mind regarding the therapeutic potential of RNAi. It's too early to make predictions," he says.

Isis is so far the only company to have succeeded in getting an antisense drug licensed - Vitravene for the treatment of skin disease - and has other antisense products in clinical trials. In contrast, the company formerly known as Ribozyme Pharmaceuticals relaunched in 2003 as Sirna Therapeutics after its most promising antisense candidates proved ineffective in clinical trials. The RNAi technology breakthrough occurred at the right time, according to Nassim Usman, senior vice-president at Sirna.

Others point out the additional concerns that antisense molecules can trigger immune reactions, being larger and 'foreign' in composition, compared with siRNA, and can be toxic. But Bennett asserts that Isis has modified its antisense products to minimize these potential problems.

NeoPharm in Illinois is maintaining a stake in both approaches. While results from animals show the overall effectiveness of RNAi to be "very much better than with antisense", according to chief scientific officer Imran Ahmad, and to require a fivefold smaller dose, the company intends to compare its lipid-based delivery system for antisense and RNA side-by-side in the clinic. 
should then take up the construct by endocytosis (see 'Express delivery', page 601). Another approach is to rely on the physiological likelihood that the construct will be taken up by the desired tissue.

Working with siRNA encased in a cationic polymer, Jianzhu Chen of the Massachussetts Institute of Technology (MIT) Center for Cancer Research has found that in mice the RNA is taken up most efficiently by lung cells following injection into the tail vein. This is probably because the lung contains the first capillary beds traversed by intravenously injected material and is the tissue with the most extensive blood supply.

And NeoPharm in Lake Forest, Illinois, is developing liposome carriers for RNAibased cancer therapy. By modifying the surface charge of a naturally occurring mitochondrial membrane lipid, cardiolipin, the company has developed a cationic liposome that is less toxic than current positively charged liposomes, according to chief scientific officer Imran Ahmad. To get the RNA to its target, NeoPharm is relying on the preferential uptake of the liposomes as a source of fat by fast-growing tumours.

Meanwhile Mirus Bio in Madison, Wisconsin, is developing a new delivery method - initially for gene therapy but eventually also for RNAi-based therapy — that targets skeletal muscle. Tested so far in animals, this involves applying a tourniquet to the leg, to maintain a high local volume of blood, followed by injection into veins in the foot. The company hopes to develop this method to treat muscular dystrophy and ischaemia by gene therapy, says Jim Hagstrom, vicepresident of scientific operations.

\section{Viral vectors}

Some researchers in the field point out that no one has yet shown chemically synthesized siRNA to work in vivo for more than a few weeks, or to be able to target a broad range of tissues. And not everyone is convinced that encasing the nucleic acid in polymers or liposomes is the best way to go.

"It'll be great to have polymers if they work, but I don't think they're efficient enough yet - viruses have learned to do this over millions of years," says Inder Verma, a geneticist at the Salk Institute for Biological Studies in San Diego, who is developing lentivirus-based expression systems for long-term RNAi in vivo. The vectors contain DNA inserts linked to a promoter recognized by RNA polymerase III, and are designed to express shRNA inside fertilized mouse eggs, creating 'knock-down' transgenic mice in just three to four weeks, with the effect lasting over many generations.

Unlike plasmids, viral vectors can introduce siRNAs into non-dividing cells such as neurons, and the lentivirus vectors have the advantage over adenovirus of being able

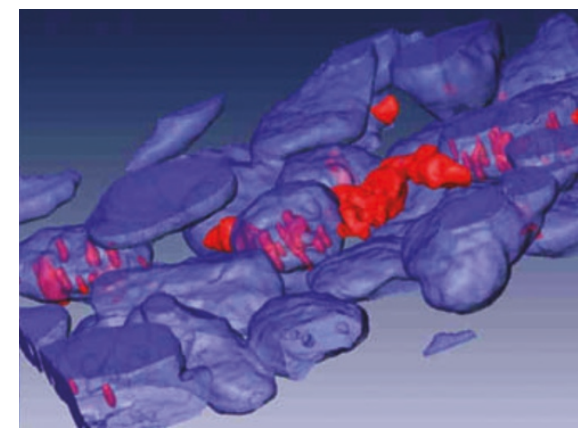

Viral shRNA transcripts (red) being exported from cell nuclei (blue).

to introduce RNAs into blood and bonemarrow cells. Luk Van Parijs and colleagues at MIT have successfully induced RNAi in haematopoietic stem cells using lentivirus vectors.

Both Verma and Van Parijs advocate the use of lentivirus vectors for pinpointing genes involved in complex and chronic diseases such as Alzheimer's disease and type 1 diabetes, and for countering their effects.

According to Verma, many viral expression systems have disadvantages in vivo because they cannot be targeted to individual tissues. Instead, they have to be injected locally, or cells need to be removed from the body, treated ex vivo and replaced. $\mathrm{He}$ also cautions that, as with siRNA, vectorexpressed shRNA has the potential to produce non-targeted effects, which could lead to side effects.

Different vectors will probably have to be developed for different tissues. Beverly Davidson, associate director at the University of Iowa Center for Gene Therapy, turned to adenovirus-associated virus (AAV) vectors in her work on a mouse model of spinocerebellar ataxia after finding it "difficult to get siRNA into tissues" in vivo, she says. The treatment entails direct injection of the AAV vector into the brain. "AAV is good at transducing in the brain, which is a well-localized organ and does not permit the virus to spread elsewhere in the body," notes Verma. AAV also has the advantage that it has already been through clinical trials for gene therapy and can be produced to a thousand-fold higher concentration than lentiviruses. Davidson sees the prospect for developing this into human therapy as "very exciting", but insists on a conservative estimate on the timeline. "If we could overcome the hurdles, then it would be reasonable to consider human trials within five years," she says.

Meanwhile, John Rossi of the City of Hope hospital in Duarte, California, is developing lentivirus-based shRNA therapy against HIV. The lentiviruses will contain DNA encoding shRNA against the HIV tat and rev genes, which are expressed early in HIV's life cycle. Stopping the virus in its tracks so early should also minimize the risk of resistance arising. Rossi hopes to get permission to test the system in AIDS patients undergoing bone-marrow transplants for AIDS-related lymphoma.

\section{Keeping safe}

Safety is paramount when it comes to using viral vectors in humans. The current generation of lentivirus vectors are "very safe", according to Rossi, as judged by animal studies and a current clinical trial for HIV using a lentiviral vector to deliver an antisense construct, conducted by VIRxSYS of Gaithersburg, Maryland. Benitec, a company in Queensland, Australia, that specializes in vector-based RNAi, may commercialize Rossi's lentiviral system if it proves successful. The firm is also supporting work on an AAV-based RNAi against hepatitis $\mathrm{C}$ virus ( $\mathrm{HCV}$ ) originating in the work of Mark Kay of Stanford University in California. HCV is an ideal target for RNAi, according to Alexander Kolykhalov, scientific director at Benitec, as it is an RNA virus and is constantly available in the cytoplasm.

AAV has only shown minor side effects in gene therapy in dogs, and in a limited trial of gene therapy for muscular dystrophy in humans, and Kay claims that these problems are "technically solvable". He also plans to use a different AAV serotype in his work. But he admits that "it's impossible to predict whether the same problems would arise".

Davidson agrees that "AAV has undergone the most evaluation and seems practical for the delivery of RNAi". But Pardridge is more critical. "With AAV and retrovirus vectors you're just rolling the dice," he says, because of the risk of insertional mutagenesis and cancer. Benitec counters that AAV is not a human pathogen, and cannot replicate or spread once inside cells. It rarely integrates into DNA, and when it does, it is at natural chromosomal breakpoints rather than transcriptional 'hotspots' where activation of potential oncogenes is likely to occur. Benitec expects an Investigational New Drug application to test the AAV-based RNAi therapy in humans "to gain approval fairly quickly" because of the previous use of the virus in trials of gene therapy. It hopes to begin clinical trials by 2006 .

There is still some way to go before RNAi's full clinical applications are defined. The current scene is unnervingly reminiscent of the excitement more than a decade ago about ribozymes and antisense (see 'A rival to antisense?', page 603). “There's a lot of hype and a lot of hope. So far I'm not convinced that RNAi will be better than antisense," says Klaus Giese, chief scientific officer at atugen in Berlin. "Everyone is waiting for a strong proof of principle in vivo." But however the therapies pan out, it is becoming clear that RNAi is already a powerful tool for probing disease pathways.

Julie Clayton is a science writer based in Bristol, UK. 